\title{
Implementation of Next Generation Mobile Service: The Context-Aware Follow-Me Service
}

\author{
Jungsook Bae ${ }^{1}$, Seungwan Ryu ${ }^{2, \star}$, JaeYong $\mathrm{Lee}^{3}$, and ByungChul Kim ${ }^{3}$ \\ ${ }^{1}$ Mobile Telecom. Research Division, ETRI, Taejon, Korea \\ ${ }^{2}$ Department of Information Systems, Chung-Ang University, Korea \\ ${ }^{3}$ School of Computer \& Communications Engineering, \\ Chungnam National University, Korea \\ jsbae@etri.re.kr, rush2384@cau.ac.kr, \\ \{jylee, bckim\}@datacom.cnu.ac.kr
}

\begin{abstract}
With the advent of mobile communication technologies, next generation mobile service is evolving toward user-centric services, in that each user can get a personalized mobile service based on his/her needs. In this paper, we propose the context-aware follow me (CAF) service as a prototype next generation user-centric mobile service. The CAF service is designed to provide optimized service based on various context information such as user's location and preference, capability of devices located around a user and characteristics of services in use. We also present an example CAF service scenario, an overall system architecture, a test-bed system and a sample CAF service flows.
\end{abstract}

\section{Introduction}

The next-generation (NG) mobile communication is expected to be a system capable to provide a variety of multimedia services such as multimedia moving pictures, movies, and television broadcasts with high-speed and high quality via the converged network of wireless and wired infrastructures [1]. In particular, with the advent of next generation mobile communication technologies such as a high-speed data rate, converged network infrastructure, appearance of new digital devices having embedded sensing capabilities and appearance of next generation mobile terminal having higher computing capabilities, advanced mobile services will be realized over whole spheres of our everyday life. Therefore, in accordance with above technological advances, next generation mobile service is evolving toward user-centric services, in that each user can get a personalized mobile service based on his/her needs [2].

In order to provide the user-centric mobile services, a best suitable service should be generated and provided to a user based on his/her current situation by collecting context information around him without restrictions on his location or time. In other words, to provide various user-centric mobile services, next generation mobile services should be able to support various advanced service

\footnotetext{
* Corresponding author.
} 
functions such as context-awareness for providing a user best suitable services based on context information around him, personalization for providing optimized services based on user's preference and needs, service mobility for providing seamless services without restrictions on location, time, device and network, and service adaptation for providing a best suitable type of service adaptively to the change of situation around a user [3].

In this paper, we propose the context-aware follow me $(C A F)$ service as a prototype next generation user-centric mobile service that can supports above functions. The CAF service is designed to provide optimized service based on various context information such as user's location and preference, capability of devices located around a user and characteristics of services in use. In this service, users can enjoy the seamless call service and the video on demand (VOD) service anywhere and anytime via best suitable device.

This paper is consisted as follows. In next section, concepts, paradigms and key features of next generation mobile service are introduced. In section 3 , design concepts and features of user-centric CAF services are proposed with example service scenarios and an overall system architecture. In section 4, a prototype test-bed system and example service flow of the CAF service is presented. Finally we conclude this paper.

\section{Next Generation Mobile Services}

With the progress of mobile communication technologies, mobile services are evolving to provide a variety of advanced services to guarantee people happiness of life [2]. The key feature of such evolutional trend of next generation mobile service is the concept of user-centric service which means that a best suitable service is provided for a user by analyzing user's needs and situational information. The concept of user-centric service is considered as a kernel paradigm of next generation mobile services and its enabling technologies are currently exploited by next generation mobile service research organizations such as Wireless World Research Forum (WWRF) [3] and Wireless World Initiative (WWI) [4]. In particular, after setting up "I" as a subject of mobile service, WWRF is trying to establish the concept of I-centric service and its enabling technologies with focusing on service requirements of "I" to support personalization, ambient awareness and adaptability. In the I-centric service environment, a personalized mobile service is provided to a user after service selection and adaptation process based on user's service requirements and context information.

In order to provide user-centric services, a best suitable service should be provided to a user after service selection and adaptation processes based on his/her current situation by collecting context information around him without restrictions on his location or time. The user-centric service should be able to provide various service features in terms of cost, data rate, maturity and application areas as follow [2]. 
- Low cost service: diverse and convenient services at low price

- High speed service: transmission of large amount of multimedia data with high data rate

- Context-Aware service: a best suitable service based on context information and situation of a user.

- Seamless service: service capable to overcome user's movement and/or changes of network environments

- Converged service: various services provided by connecting or combining mobile services with other services such as broadcasting and other information technologies.

- Personalized service: a tailored service to the user's expectation in accordance with diverse demand and desire and personalized life style.

- Virtual reality service: services that are true to nature provided by various user friendly interfacing methods.

- Safety service: services for keeping security and privacy.

In order to fulfill user-centric services having above service features, functions of context-awareness, personalization, service mobility and adaptation should be supported.

\section{The Context Aware Follow-Me Service}

\subsection{Concepts and Features}

A number of context-aware computing and communication concepts and applications have been proposed by research organizations and companies $[5,6]$. However, most of such context-aware services were not suggested as mobile services in next generation [7]. For example, [6] introduces recent research and development activities on context-aware communications with focusing on how changes on individual context initiate and facilitate people's interaction with one another.

In this paper, we propose the context aware follow-me (CAF) service developed as a prototype next generation user-centric context-aware mobile service. CAF service is designed for providing optimal services using best suitable devices based on user's location and preference, capability of available devices and characteristics of services in use.

Key features of the CAF service are

- Context awareness: inference of current service availability using collected context information such as user's location, device availability and capability of devices exist in close proximity of a user.

- Service mobility: seamless transfer of on-going service from a device to other selected device based on service environment where a user is located and user's device preference.

- Service personalization: provisioning of service environment user prefer using context information such as user profile, user's preferences, and other environmental information. 
Tom is driving a car on the way home. He takes French lesson using the mobile foreign language lesson service. Since he is driving, he takes lesson only in audio sound mode. After arriving home, Tom gets out of the car, but the lesson is continued via his mobile terminal with giving audio and video mode services simultaneously. As soon as he enters living room the lesson service is shifted to a TV. After finishing French lesson, Tom begins taking a bath. When he is in bath room, Jain calls him, and the call is forwarded to a smart mirror equipped in bath room only with voice mode. When the bath is over, the call is shifted to his mobile terminal with giving voice and video mode simultaneously.

Fig. 1. An example CAF service scenario

- Service adaptation: adaptation of service data format and quality according to capability of the selected device and network.

A possible service scenario of the CAF service is given below.

\subsection{System Architecture}

As a prototype service of next generation context-aware service, the CAF service is provided within a local domain via interfacing with other remote domains. Each domain is consisting of several elements such as a domain server, a session control server, a location server, location management systems, a media adaptation server and smart devices. Functions of each system elements are.

- A domain server identifies and authorizes a user, collects context information of a user, gathers information related to other devices, and controls session mobility. Examples are the home server, the office server, the car server.

- A session management server manages session connections or hands over a session connection to a selected intelligent device.

- A location management system detects the user's current location.

- A location server identifies user's current location.

- A media adaptation server trans-codes media to be suitable to the selected intelligent device and, vise versa.

- A smart device has communication capability with system elements in domain and multimedia service support capability.

- A mobile terminal can support multi radio access technologies such as CDMA, WLAN and WPAN.

The overall system architecture for the CAF service is given in figure 2 .

Generic operational rules and sequences of the CAF service under the given architecture are as follow.

1. A user enters a new domain such as a car, a house or an office.

2. A (local) domain server collects device related information device such as location, device capability and availability periodically using the device discovery protocol. 


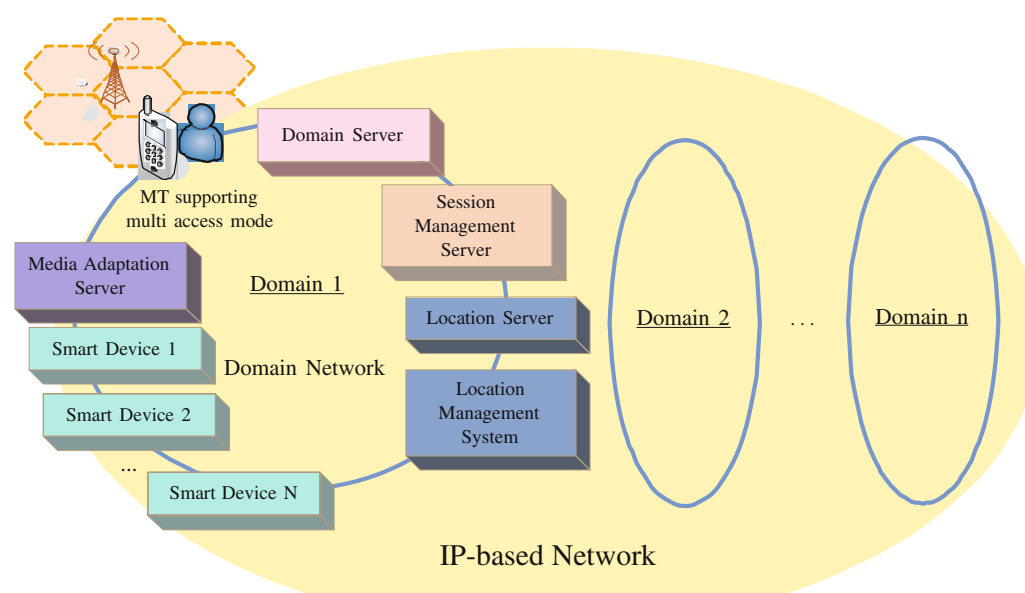

Fig. 2. An overall system architecture for CAF service

3. The domain server identifies and authorizes the user.

4. Location management systems and a location server keep track of location of the authorized user.

5. The domain server collects users current context information such as user location, using service, user profile and user preferences.

6 . When the user is under calling with someone or getting media streaming service, the domain server selects the best suitable device based on users current location, users preferences, device information and service characteristics.

7. The domain server manages session control server to transfer the user's current session connections to the selected device

8. The domain server manages the media adaptation server which transforms media code to be suitable for the selected device.

\subsection{The Test-Bed System for CAF Service}

In order to investigate feasibility of the CAF service, especially for the example CAF scenario presented in figure 1, we developed a test-bed system. As shown in figure 3 , the test-bed system consists of two domains, a car domain and a home domain, in addition to a mobile terminal that can roam those two domains. The car domain is consisting of a car server, location systems, a location server, a media adaptation server and a car A/V system which has communication and computing capability. All devices in the car domain are assumed to be connected via short-range communications. In this test-bed, we use WLAN for communications among devices. The home domain is consisting of a home server, location systems, a location server, a media adaptation server and smart devices such as an intelligent TV, audio and smart mirror are assumed to have communication and computing capability. In addition, such devices are also assumed to be connected via WLAN similar to the devices in the car domain. The home domain 


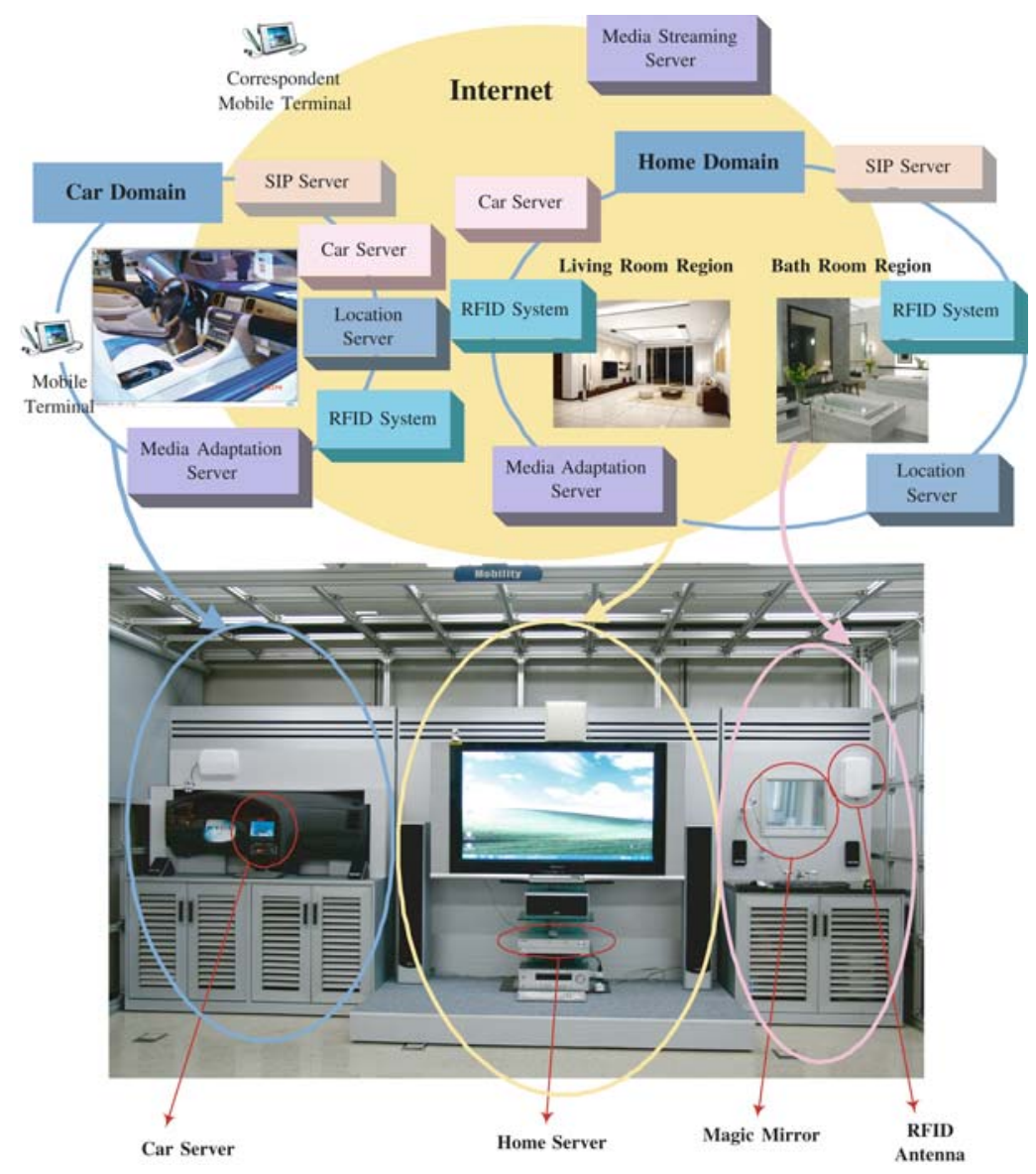

Fig. 3. Test bed system for CAF service

is divided into two sub-regions, the living room region and the bath room region. For tracking and recognition of user location, a passive type RFID tag is attached on a user, and 915Mhz RFID readers are installed in several parts of each domain. In this test-bed, the service mobility across a range of devices in various domains is supported by the session initiation protocol (SIP).

\subsection{Service Flow in Test-Bed System}

Because of page limitation, we give readers a sample CAF service flows for a specific situation instead of giving a complete set of CAF service flow for the example CAF service scenario shown in figure 1. In detail, we choose a specific situation when the user gets off the car and enters home, and show how the CAF service is provided in this situation. We describe service flows among service elements such as a mobile terminal (MT), a home server (HS), a location server (LS), a media adaptation server (MAS), a media streaming server (MSS), a 


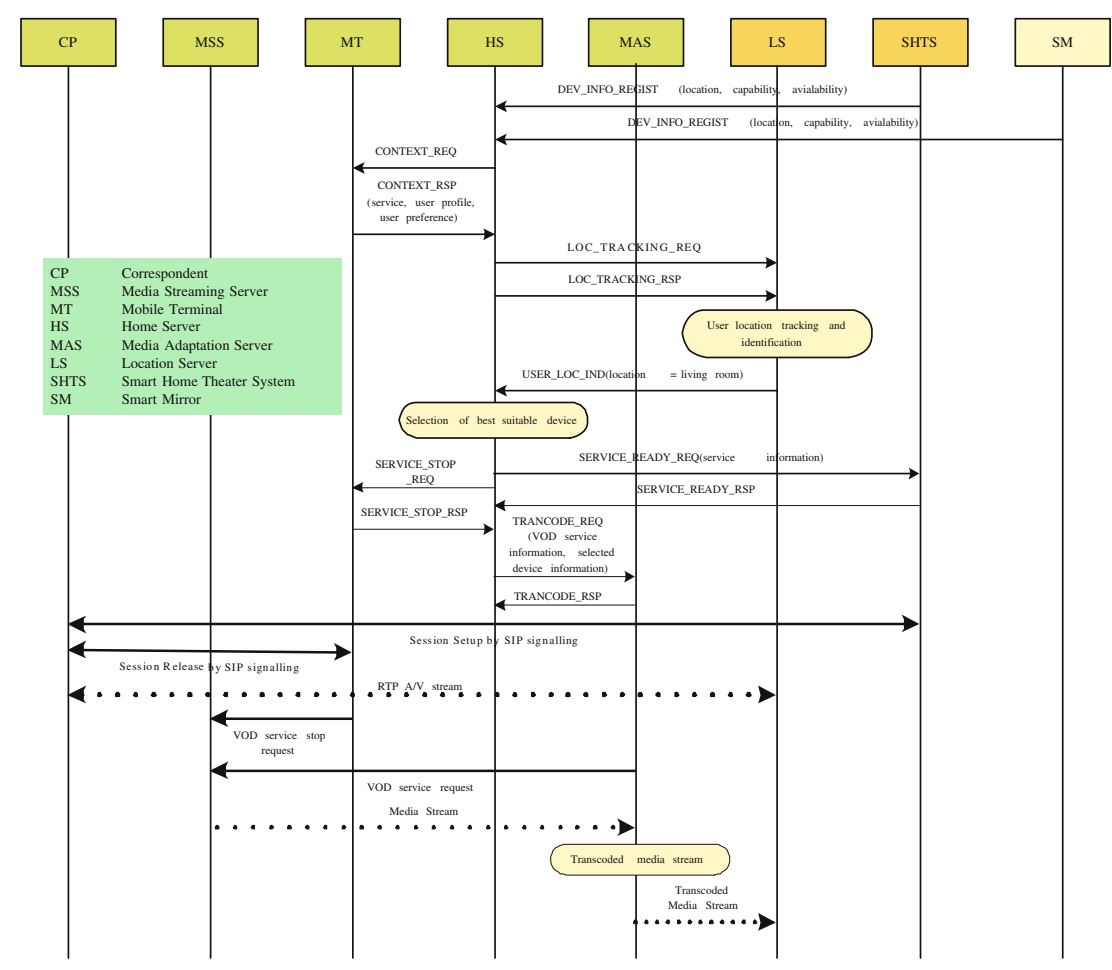

Fig. 4. CAF Service Flow in test bed system

smart home theater system (SHTS), and a correspondent (CP) who is a person connected with the user's terminal through SIP protocol. In the chosen situation, we assume that the user enters a home domain while using both of VoIP call service and the VOD service via his mobile terminal. Figure 4 describes how service flows are implemented among service elements in the test-bed system to maintain the CAF service seamlessly in this situation.

Each domain server collects device related information such as device location, device capability and availability from service devices exist in the same domain. When a new user is identified, a domain server (the home server (HS) in this case) requests context information of the identified user stored in the former domain server (the mobile terminal (MT) of the user or the car server in this case). Upon receiving such request from HS, the mobile terminal delivers context information such as services in use, user profile and preferences, and other context information. At the same time, HS requests location tracking information of the identified user to the location server (LS). Since LS detects the user enters living room, it informs the current location and location tracking information of the user to HS. Then, based on collected context information such as user's current location, user's profile and preference, device information and service information, HS selects the smart home theater system (SHTS) as the 
best suitable service device. Then, HS delivers service information to SHTS to make it ready for service. Consequently, HS requests MT to suspend services in use. At the same time, HS delivers information about service and selected device as well as requests for streaming media code transformation to the media adaptation server (MAS) to give SHTS optimal service environment. SHTS sets up a session with correspondent $(\mathrm{CP})$ via SIP signaling, and MT releases the session with correspondent by SIP signaling and requests the media streaming server (MSS) to stop VOD service. At the same time, MAS requests MSS to resume of VOD service to SHTS. Finally, SHTS begins to serve the identified user.

\section{Conclusion}

In this paper, we investigate evolutional trend, concepts and key features of next generation mobile services. We then introduce the context-aware follow me (CAF) service designed to support context awareness, service mobility, service personalization, service adaptation. Finally, we give readers an example CAF service scenario, an overall system architecture, a test-bed system and a sample service flows. We hope that our research activities and results on next generation mobile services, especially the CAF service presented in this paper, will play important role in developing core technologies of next generation mobile communication systems.

\section{References}

1. S. Ryu and D. Oh and G. Shin and K. Han and S. Hwang and S. Park: "Research Activities on the Next Generation Mobile Communications and Services in Korea," IEEE Communicaions Magazine, 43(9), pp. 122-131, September, 2005.

2. Next Generation Mobile Communication Forum: "Rainbow Vision," http://www.ngmcforum.org/ngmc2/main.html.

3. S. Arbanowski and P. Ballon and K. David and O. Droegehorn and H. Eertink and W. Kellerer and H. Kranenburg and K. Raatikainen and R. Popescu-Zeletin: "I-centric Communications: Personalization, Ambient Awareness, and Adaptability for Future Mobile Services," IEEE Communicaions Magazine, 42(10), pp. 63-69, September, 2004.

4. Wireless world initiative: "Mobi Life: Life goes on," http://www - wireless-worldinitiative.org/.

5. G. Chen and D. Kotza: "A survey of context-aware mobile computing research," Technical Report, TR2000-381, Department of Computer Science, Dartmouth College, May, 2000.

6. B. Schilit and D. Hilbert and J. Trevor: "Context-Aware Communication," IEEE Wireless Communicaions Magazine, 9(5), pp. 2-10, October, 2002.

7. J. Kim and J. Bae and S. Yoon and J. Ha and G. Sihn: "Evolution of ContextAware Services in Next Generation Mobile Communication," WG contributions of 13th WWRF Meeting, March, 2005. 